\title{
Combined high resolution chromatographic techniques (FPLC and HPLC) and mass spectrometry-based identification of peptides and proteins in Grana Padano cheese*
}

\author{
P Ferranti ${ }^{1}$, E Itolli ${ }^{1}$, F Barone ${ }^{1}$, A Malorni ${ }^{2}$, G Garro ${ }^{1}$, \\ P Laezza ${ }^{1}$, L Chianese ${ }^{1}, \mathrm{~F}$ Migliaccio ${ }^{1}$, V Stingo ${ }^{1}, \mathrm{~F}$ Addeo ${ }^{3}$ \\ ${ }^{1}$ Dipartimento di Scienza degli Alimenti dell'Università degli Studi di Napoli Federico II, \\ I-80055 Portici, ${ }^{2}$ Servizio di Spettrometria di Massa , CNR, Via Pansini 5, I-80131 Napoli; \\ ${ }^{3}$ Istituto Sperimentale Lattiero-Caseario, Via A Lombardo 11, I-20075 Lodi, Italy
}

Summary - The $0.2 \mathrm{~mol} / \mathrm{L}$ trisodium citrate soluble $\mathrm{N}$ fraction of 14 - and 38-month-old Grana Padano (GP) cheese diafiltered through 3 - and 10-kDa cut-off membranes gave two fractions containing peptides with a molecular mass lower than $3 \mathrm{kDa}$, and higher than $10 \mathrm{kDa} .91$ and 38 oligopeptides lower than $3 \mathrm{kDa}$ were identified by using a combination of fast-atom bombardment/mass spectrometry and Edman degradation in the 14- and 38-month-old GP cheese samples respectively. Peptides higher than $10 \mathrm{kDa}$ were submitted to preparative fractionation on Mono Q column with a stepwise ionic strength gradient and then by reverse phase-HPLC on a C4 column. Some native caseins and large-derived peptides were identified by using a combination of electrospray mass spectrometry and Edman degradation.

Grana Padano / casein proteolysis / oligopeptide / mass spectrometry

Résumé - Identification de peptides et protéines dans le fromage Grana Padano à l'aide de techniques chromatographiques haute résolution (FPLC et HPLC) couplées à la spectrométrie de masse. La matière azotée soluble dans le tampon citrate $0,2 \mathrm{~mol} / \mathrm{L}$ à $\mathrm{pH} 8,0$ de deux échantillons de 14 et 38 mois de Grana Padano, un fromage italien à pâte dure, a été diafiltrée sur membranes à porosité de 3 et $10 \mathrm{kDa}$. Deux fractions ont été recueillies, contenant des peptides de masse moléculaire inférieure à $3 \mathrm{kDa}$ et supérieure à $10 \mathrm{kDa}$. La fraction inférieure à $3 \mathrm{kDa}$ a été fractionnée par chromatographie liquide haute performance (CLHP) en phase inverse sur colonne C18 et les peptides identifiés en combinant la spectrométrie de masse à bombardement rapide et la dégradation d'Edman. La plupart des 91 peptides identifiés dans cette fraction A du fromage de 14 mois provenaient des

\footnotetext{
* Presented as an oral communication at the IDF Symposium 'Ripening and Quality of Cheeses', Besançon, France, February 26-28, 1996.
} 
caséines $\alpha_{\mathrm{s1}}$ (44 peptides) et $\beta$ (32 peptides). La majorité de ces derniers présentaient une origine commune dans la région 1-105/107 de la caséine $\beta$. Trente peptides résultaient de la dégradation du peptide $\alpha_{t 1}$ - CN (f1-79) formé par action de la plasmine sur la caséine $\alpha_{s 1}$. Treize peptides provenaient de la caséine $\alpha_{s 2}$ et un seul de la para- $\kappa$-caséine. Trente-huit peptides ont été identifiés dans la fraction inférieure à $3 \mathrm{kDa}$ du fromage de 38 mois (dont 21 peptides identifiés dans le fromage jeune) : 8 issus de la caséine $\beta, 27$ de la caséine $\alpha_{\mathrm{s} 1}, 1$ de la caséine $\alpha_{\mathrm{s} 2}$ et 2 de la para- $\kappa-C N$. La fraction supérieure à $10 \mathrm{kDa}$ a été soumise à un fractionnement sur colonne Mono $\mathrm{Q}$, puis à une séparation par CLHP en phase inverse sur colonne C4. Les peptides ont été identifiés en combinant la spectrométrie de masse à l'électrospray et la dégradation d'Edman. Les résultats concernant une fraction chromatographique sont présentés : les variants B et C de la caséine $\alpha_{s 1}$ et plusieurs fragments de grosse taille tels que le peptide $\alpha_{51}$-CN-I ont été identifiés. Les résultats montrent que les différentes techniques de spectrométrie de masse permettent de caractériser, mieux que d'autres techniques, la nature des peptides issus des caséines, quelle que soit leur taille.

\section{Grana Padano / protéolyse/ peptide/ spectrométrie de masse}

\section{INTRODUCTION}

The ripening of a hard cheese such as the Italian AOC Grana Padano (GP) cheese requires at least 1 year during which, among other modifications, the extensive enzymatic hydrolysis of caseins takes place. A general mechanism of casein degradation in cheese has been suggested, implying the intervention of starter and milk proteinases and rennet in the formation of primary peptides, and peptidases in the refining of the primary products into decreasing-size peptides and free amino acids (FAAs) (Grappin et al, 1985). The $\mathrm{N}$ components of cheese consist of $\mathrm{pH} 4.6$ insoluble native caseins and derived high-molecular mass (HMM) peptides, soluble medium and low molecular mass peptides, and free amino acids. Knowledge has been accumulated on the specificity of plasmin (Andrews, 1978a, b ; Le Bars and Gripon, 1989, 1993), chymosin (Visser, 1993; McSweeney et al, 1993), whereas little information is available on proteinases and peptidases from thermophilic lactic acid bacteria (Pritchard and Colbear, 1993) such as $L$ helveticus, the species dominating in the natural 'whey' starter used for GP manufacture (Bottazzi et al, 1996). The cleavage sites in $\beta-\mathrm{CN}$ by the cell envelope proteinase from $L$ helveticus were the same as those hydrolyzed by the lactococcal $\mathrm{P}_{\mathrm{r}}$-type proteinase (Zevaco and Gripon, 1988); others were different, although there appear to be several different specificity types among the proteinases from this species (Khalid et al, 1991). The prevailing knowledge on the soluble peptides of hard cheeses is limited to the $12 \%$ trichloracetic soluble and insoluble peptides of Parmigiano Reggiano cheese (Addeo et al, 1992, 1994), the phosphopeptides from Comté cheese (Roudot-Algaron et al, 1994), and the water soluble peptides of Cheddar cheese (Singh et al, 1995).

We are attempting to describe the proteolysis of GP cheese on a molecular basis. Our present knowledge concerns a variety of HMM-plasmin-mediated peptides from $\beta$-, and to a lesser extent $\alpha_{\mathrm{s} 1}-\mathrm{CN}$, whose origin has been established either by immunoblotting with polyclonal antibodies against single casein fractions or by direct sequencing of a few isolated peptides (Addeo et al, 1995). In addition to this, in GP cheese there were a lot of $\mathrm{pH} 4.6$ soluble phosphopeptide (CPP) components which were degraded into shorter peptides (Ferranti et al, 1997). However, since CPPs represent only a secondary fraction among the wider $\mathrm{pH} 4.6$ soluble $\mathrm{N}$ cheese fraction, containing the non-phosphorylated peptides components, a 
procedure was assayed to isolate and characterize the main peptides.

Our analytical approach is based on: i) the use of a preliminary separation of the $\mathrm{N}$ cheese components according to the molecular size by diafiltration through membranes with cut-off of 3 and $10 \mathrm{kDa}$; ii) the further preparative fractionation by HPLC or FPLC to obtain single pure components or a mixture of components; and iii) the identification of peptides by mass spectrometry. In this work, oligopeptides with a molecular mass lower than $3 \mathrm{kDa}$ in 14- and 38-monthold GP cheese samples have been characterized with a few HMM-peptides from the 14-month-old sample, by using a combination of fast atom bombardment (FAB)/ or electrospray(ES)/mass spectrometry (MS) and Edman degradation.

\section{MATERIALS AND METHODS}

\section{Cheese samples and fractionation of cheese nitrogen}

Two samples of Grana Padano cheese (GP) after 14 and 38 months of ripening supplied by the 'Consorzio di Tutela del formaggio Grana Padano' (Milan, Italy) were used in this study. Grated cheese was freeze-dried and extracted with diethyl ether in a Soxhlet apparatus. Cheese powder $(1 \mathrm{~g})$ was suspended in $20 \mathrm{~mL} 0.2 \mathrm{~mol} / \mathrm{L}$ trisodium citrate buffer ( $\mathrm{pH} 8$ ), then kept in an ultrasonic bath (Mod T 460, Carlo Erba Reagenti, Milan) for $15 \mathrm{~min}$, then shaken for an additional $30 \mathrm{~min}$ and left to stand $1 \mathrm{~h}$ at $4{ }^{\circ} \mathrm{C}$. After centrifugation at room temperature for $20 \mathrm{~min}$ at $4000 \mathrm{~g}$, the fat layer was carefully removed with a spatula. The resulting soluble $\mathrm{N}$ cheese fraction was filtered on Whatman paper $3 \mathrm{MM}$ and then ultrafiltered using 3 - and $10-\mathrm{kDa}$ cut-off membranes (Centripep, Amicon Division, Beverly, MA, USA). Each retentate was diluted with $20 \mathrm{~mL}$ citrate solution and ultrafiltered again. The operation was repeated three times giving rise, after pooling, to three fractions containing peptides with a molecular mass lower than $3 \mathrm{kDa}$, between 3 and $10 \mathrm{kDa}$ and higher than $10 \mathrm{kDa}$. Each fraction was concentrated three times in a centrifuge operating under vacuum
(SpeedVac, Concentrator SVC 100H, SVPT srl, Rome, Italy) and stored at $-20{ }^{\circ} \mathrm{C}$ until further analysis.

\section{Reverse phase HPLC of peptides with a molecular mass lower than $3 \mathrm{kDa}$}

The permeate containing peptides with a molecular mass lower than $3 \mathrm{kDa}$ was fractionated by reverse phase (RP)-HPLC on a Kontron equipment (Kontron Instruments, Milan, Italy) consisting of two Model 420 pumps, a Rheodyne sample injector ( $250 \mu \mathrm{L}$ loop) and a Model 491 solvent programmer. Peptide detection was carried out at $220 \mathrm{~nm}$ using a Kontron variablewavelength detector (Model 430). Reverse phase columns $\mu$-Bondapak $\mathrm{C} 18,250 \times 4.6 \mathrm{~mm}$ and $250 \times 10 \mathrm{~mm}$ (Waters Associates, Milford, MA, USA) were used for analytical and semi-preparative purposes. Solvent A was $0.1 \%$ trifluoroacetic acid (TFA) in water. Solvent B was $0.1 \%$ TFA in acetonitrile. The flow was of $1 \mathrm{~mL} / \mathrm{min}$. The gradient was: $\mathrm{t}=0 \mathrm{~min}, \% \mathrm{~B} 0 ; \mathrm{t}=5 \mathrm{~min} \% \mathrm{~B}, 0$; $\mathrm{t}=34 \mathrm{~min}, \% \mathrm{~B} 29 ; \mathrm{t}=35 \mathrm{~min}, \% \mathrm{~B} 38 ; \mathrm{t}=43 \mathrm{~min}$, $\%$ B $48.250 \mathrm{~mL}$-volumes were injected into the column and the peptide fractions corresponding to each chromatographic peak collected, pooled and dried by centrifugation under vacuum. The operation was repeated ten times to obtain enough peptide material to characterize peptides by FAB/MS.

\section{Identification of peptides by fast atom bombardment mass spectrometry (FAB/MS)}

FAB mass spectra were recorded on a VG Analytical ZAB 2 SE double-focusing mass spectrometer (VG Instruments, Manchester, UK) fitted with a VG cesium gun operating at $25 \mathrm{keV}$ $(2 \mu \mathrm{A})$ in the mass range $300-3000 \mathrm{Da}$. The spectra were recorded on UV direct print paper and counted manually. The dried HPLC fractions were dissolved in $1 \mathrm{~mol} / \mathrm{L}-\mathrm{HCl}$ and loaded onto a glycerol-thioglycerol-coated probe tip. The peptide components were identified on the basis of the molecular mass value and the $\mathrm{N}$-terminal sequence as described previously (Addeo et al, 1992), taking into account the amino acid sequences of $\alpha_{s_{1}}, \beta$-, and $\alpha_{s 2}$-casein reported by Swaisgood (1993) taking into account the corrections made by different authors. 


\section{Chromatographic fractionation of peptides with molecular mass above $10 \mathrm{kDa}$}

The fraction containing the oligopeptides with a molecular mass higher than $10 \mathrm{kDa}$ was further fractionated by a fast protein liquid chromatography on a Mono Q (HR 10/10, Pharmacia, Uppsala, Sweden) anion exchange column equilibrated in the $20 \mathrm{mmol} / \mathrm{L}$ monosodium phosphate, $\mathrm{pH} 7.0$ buffer and operating at room temperature at $1.0 \mathrm{~mL} / \mathrm{min}$. A linear gradient from $0-0.32 \mathrm{~mol} / \mathrm{L} \mathrm{NaCl}$ was used. Fractions corresponding to each peak were pooled, dialyzed against distilled water and freeze dried. Single fractions were further separated by HPLC on a C4 column according to the procedure of Ferranti et al (1995).

\section{Identification of peptides by electrospray mass spectrometry (ES/MS)}

The identity of the single peptides was established by a combination of electrospray (ES)-mass spectrometry (MS) and Edman degradation. In brief, molecular masses were assigned to the corresponding peptides along the sequence of the four casein fractions by using a suitable computer program developed in our laboratory (F Barone, unpublished data). The assignments were confirmed by submitting the peptide fractions to at least two steps of manual Edman degradation, followed by ES/MS analysis of the truncated species, in order to obtain the necessary $\mathrm{N}$-terminal sequence information.

\section{RESULTS}

\section{HPLC of Grana Padano low molecular mass peptides}

A fraction containing soluble low molecular mass $\mathrm{N}$ forms was isolated by diafiltration through a 3-kDa cut-off membrane starting from a citrate buffer solution of freeze-dried and defatted GP cheese. The RP-HPLC profiles of the 14- and 38-month-old cheese samples are shown in figure 1 . They were

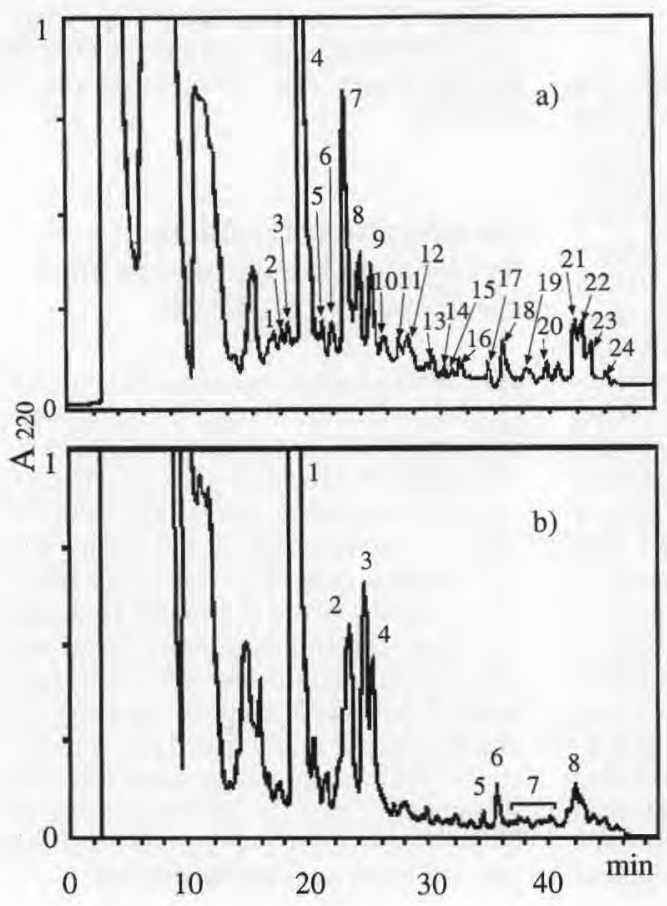

Fig 1. Reversed-phase HPLC profile of oligopeptides with molecular mass lower than $3 \mathrm{kDa}$ of 14- (a) and 38-month-old (b) Grana Padano cheese, showing the peak or group of peaks collected and identified.

Profil CLHP en phase inverse des peptides de masse moléculaire inférieure à $2 \mathrm{kDa}$ issus de Grana Padano de 14 (a) et 38 mois (b), montrant les pics, ou groupes de pics, collectés et identifiés. 
similar to those of Parmigiano-Reggiano (PR) cheese (Addeo et al, 1992; Fox and McSweeney, 1994), also belonging to the same class of AOC hard cheese. The group of peaks eluted in the final part of the chromatogram was quantitatively more important in the cheese of lower age. The two samples of 14- and 38-month-old GP cheese were examined in detail for structural characterization.

\section{The nature of oligopeptides in 14-month-old Grana Padano cheese}

About 30 peaks were collected from the fractionation by HPLC of the 14-month-old cheese sample, as shown in figure 1a. After drying, peptides were subjected to molecular mass determination by FAB/MS. By using a suitable software program for the identification of peptides through the molecular mass, many possible peptides were found for each experimental mass value, as shown in table I, concerning the peak $n 9$ of the HPLC separation shown in figure 1a.
The results indicated that the molecular mass determination alone did not allow peptides singly or in mixture be unambiguously identified. Therefore, in order to improve the procedure of identification, additional structural information was searched through the $\mathrm{N}$-terminal residue determination; however, a mixture of $\mathrm{N}$-terminal residues was obtained owing to the presence of several peptides in the mixture. This made the automated sequencing of peptides in mixture ineffective for the peptide identification. This problem was overcome through the determination of the molecular mass by $\mathrm{FAB} / \mathrm{MS}$ on the truncated peptides in mixture after each consecutive Edman degradation step. However, the determination of the $\mathrm{N}$-terminal residue only reduced the number of the possible peptides among those having similar molecular mass, as reported in table I. A reliable identification of the peptide components in mixture was obtained through the determination of two or three $\mathrm{N}$-terminal residues in addition to the molecular mass of the native peptide. Following this procedure, the oligopeptides having

Table I. Identification steps by FAB/MS and Edman degradation of the peptides occurring in the HPLC peak number 9 relative to 14 -month-old Grana Padano cheese.

Étapes d'identification par FAB/MS et dégradation d'Edman des peptides apparaissant dans le pic 9 issus d'un Grana Padano de 14 mois.

\begin{tabular}{|c|c|c|c|c|c|c|}
\hline $\begin{array}{l}\text { Molecular } \\
\text { mass of } \\
\text { single } \\
\text { peptides, } \\
\text { m/z }\end{array}$ & $\begin{array}{l}\text { Number of } \\
\text { casein } \\
\text { peptides } \\
\text { having } \\
\text { similar } \\
\text { mol mass } a\end{array}$ & $\begin{array}{l}\text { Molecular } \\
\text { mass after } \\
\text { a first } \\
\text { Edman } \\
\text { cycle }^{b}\end{array}$ & $\begin{array}{l}\text { Number of } \\
\text { peptides } \\
\text { having } \\
\text { similar mol } \\
\text { mass and } \\
\text { N-terminal } \\
\text { residue }\end{array}$ & $\begin{array}{l}\text { Molecular } \\
\text { mass after } \\
\text { the second } \\
\text { Edman } \\
\text { cycle }^{\text {a }} \\
\text { Number of } \\
\text { peptides }\end{array}$ & $\begin{array}{l}\text { mol mass } \\
\text { and two } \\
N \text {-terminal } \\
\text { residues } \\
\text { Identity of } \\
\text { peptides }\end{array}$ & \\
\hline 662 & 10 & 548 (-Asn) & 1 & 435 (-Leu) & 1 & $\alpha_{s 1}(19-23)$ \\
\hline 758 & 12 & 644 (-Asn) & 2 & 515 (-Glu) & 1 & $\alpha_{s 1}(17-22)$ \\
\hline 879 & 19 & 792 (-Ser) & 2 & 764 (-Tyr) & 1 & $\kappa(37-43)$ \\
\hline 979 & 21 & 850 (-Glu) & 2 & 737 (-Leu) & 1 & $\beta(44-51)$ \\
\hline 1054 & 13 & $955(-\mathrm{Val})$ & 2 & 841 (-Asn) & 1 & $\alpha_{s 1}(37-45) 1 P$ \\
\hline 1059 & 19 & 972 (-Ser) & 3 & 901 (-Ala) & 1 & $\alpha_{s 1}(115-123)$ \\
\hline
\end{tabular}

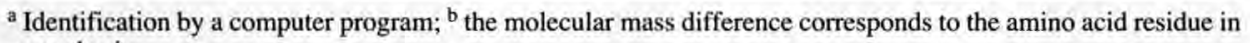
parenthesis.

${ }^{a}$ Identification effectuée par un programme informatique ; ${ }^{\mathrm{b}}$ la différence de masse moléculaire correspond au résidu d'acide aminé entre parenthèses. 
Table II. Oligopeptide components from a 14-month-old Grana Padano cheese sample found in the fractions separated by HPLC on a C18 column (fig 1a).

Peptides provenant d'un échantillon de Grana Padano de 14 mois, identifiés dans les fractions obtenues en CLHP sur colonne C18 (fig 1a).

\begin{tabular}{|c|c|c|c|c|c|}
\hline \multirow[b]{2}{*}{$\begin{array}{l}\text { HPLC } \\
\text { peak }\end{array}$} & \multirow[b]{2}{*}{$\mathrm{MH}^{+}$} & \multicolumn{2}{|c|}{$\begin{array}{l}\mathrm{MH}^{+} \text {after Edman } \\
\text { cycle }\end{array}$} & \multirow[b]{2}{*}{$\begin{array}{l}N \text {-terminal } \\
\text { sequence }\end{array}$} & \multirow[b]{2}{*}{ Peptide } \\
\hline & & $I$ & III & & \\
\hline 1 & 746 & 689 & 590 & Gly-Val & $\beta(94-100)$ \\
\hline 2 & 1048 & 881 & 782 & SerP-Val & $\alpha_{\mathrm{s} 1}(75-82) 1 \mathrm{P}$ \\
\hline \multirow[t]{2}{*}{3} & 921 & 754 & 683 & SerP-Ala & $\alpha_{\mathrm{sl}}(115-121) 1 \mathrm{P}$ \\
\hline & 609 & 494 & 423 & Asp-Ala & $\alpha_{\mathrm{s} 1}(157-162)$ \\
\hline \multirow[t]{3}{*}{4} & 659 & 546 & 489 & Ile-Gly & $\alpha_{\mathrm{sl}}(136-141)$ \\
\hline & 609 & 494 & 366 & Asp-Lys & $\beta(47-51)$ \\
\hline & 589 & 460 & 361 & Glu-Val & $\alpha_{s}(68-72)$ \\
\hline 5 & 593 & 464 & 349 & Glu-Asp & $\alpha_{\mathrm{sl}}(50-54)$ \\
\hline \multirow[t]{4}{*}{6} & 920 & 757 & 700 & Tyr-Leu & $\kappa(38-47)$ \\
\hline & 763 & 634 & 519 & Glu-Asp & $\alpha_{s 1}(55-60)$ \\
\hline & 761 & 632 & 519 & Glu-Leu & $\alpha_{\mathrm{s1}}(39-45)$ \\
\hline & 720 & 720 & 720 & -- & $\beta(46-51)$ \\
\hline \multirow[t]{2}{*}{7} & 850 & 737 & 609 & Leu-Gln & $\beta(45-51)$ \\
\hline & 841 & 712 & 599 & Glu-Leu & $\alpha_{11}(39-45) 1 P$ \\
\hline \multirow[t]{3}{*}{8} & 955 & 840 & 712 & Asp-Lys & $\beta(47-54)$ \\
\hline & 758 & 644 & 515 & Asn-Glu & $\alpha_{\mathrm{sl}}(17-22)$ \\
\hline & 698 & 627 & 528 & Ala-Val & $\alpha_{\mathrm{s} 2}(116-122)$ \\
\hline \multirow{6}{*}{9} & 1059 & 972 & 901 & Ser-Ala & $\alpha_{\mathrm{s} 1}(115-123)$ \\
\hline & 1054 & 955 & 841 & Val-Asn & $\alpha_{\mathrm{s}}(37-45) 1 \mathrm{P}$ \\
\hline & 979 & $850(985)$ & $737(872)$ & Glu-Leu & $\beta(44-51)$ \\
\hline & 879 & $792(927)$ & 764 & Ser-Tyr & $\kappa(37-43)$ \\
\hline & 758 & 644 & 515 & Asn-Glu & $\alpha_{s 1}(17-22)$ \\
\hline & 662 & 548 & 435 & Asn-Leu & $\alpha_{s 1}(19-23)$ \\
\hline \multirow[t]{2}{*}{10} & 1015 & $887(1022)$ & 759 & Gln-Lys & $\alpha_{\mathrm{s1}}(131-139)$ \\
\hline & 856 & 742 & 643 & Asn-Val & $\beta(7-14)$ \\
\hline \multirow[t]{4}{*}{11} & 856 & 742 & 643 & Asn-Val & $\beta(7-14)$ \\
\hline & 786 & $623(758)$ & 645 & Tyr-Leu & $\alpha_{s 2}(179-184)$ \\
\hline & 632 & 503 & 390 & Glu-Leu & $\beta(2-6)$ \\
\hline & 584 & 456 & 357 & Lys-Val & $\alpha_{s 1}(105-109)$ \\
\hline \multirow[t]{4}{*}{12} & 975 & 874(1009) & 746 & Thr-Lys & $\alpha_{s}(198-205)$ \\
\hline & 937 & 836 & 708 & Thr-Gln & $\beta(78-86)$ \\
\hline & 788 & 632 & 503 & Arg-Glu & $\beta(1-6)$ \\
\hline & 746 & 659 & 602 & Ser-Gly & $\alpha_{\mathrm{sl}}(161-166)$ \\
\hline \multirow[t]{4}{*}{13} & 869 & 812 & 699 & Gln-Leu & $\alpha_{\mathrm{sl}}(10-17)$ \\
\hline & 831 & 703 & 575 & Gln-Lys & $\alpha_{\mathrm{s} 2}(172-178)$ \\
\hline & 703 & 575 & 428 & Lys-Phe & $\alpha_{\mathrm{s} 2}(173-178)$ \\
\hline & 520 & 423 & 295 & Pro-Gln & $\alpha_{82}(177-180) /$ \\
\hline \multirow[t]{2}{*}{14} & 1150 & 994(1129) & $831(966)$ & Arg-Tyr & $\alpha_{s 2}(170-178)$ \\
\hline & 933 & $862(997)$ & $749(884)$ & Ala-Leu & $\alpha_{\mathrm{s} 2}(175-182)$ \\
\hline \multirow[t]{4}{*}{15} & 1126 & $997(1132)$ & $884(1019)$ & Glu-Leu & $\beta(44-52)$ \\
\hline & 1020 & 883 & 755 & His-Gln & $\alpha_{s 1}(8-16)$ \\
\hline & 998 & 941 & 828 & Gly-Leu & $\alpha_{\mathrm{s} 1}(10-18)$ \\
\hline & 965 & 852 & 755 & Ile-Pro & $\beta(74-82)$ \\
\hline \multirow[t]{2}{*}{16} & 1227 & 1098 & 969 & Glu-Glu & $\beta(4-14)$ \\
\hline & 1126 & $997(1132)$ & $884(1019)$ & Glu-Leu & $\beta(44-52)$ \\
\hline
\end{tabular}




\begin{tabular}{|c|c|c|c|c|c|c|}
\hline \multirow{8}{*}{17} & 1099 & 970 & \multicolumn{2}{|l|}{871} & Glu-Val & $\alpha_{\mathrm{sl}}(14-22)$ \\
\hline & 1079 & 965 & \multicolumn{2}{|l|}{852} & Asn-Leu & $\beta(73-82)$ \\
\hline & 755 & 698 & \multicolumn{2}{|l|}{585} & Gly-Leu & $\alpha_{\mathrm{s} 1}(10-16)$ \\
\hline & 1427 & $1299(1434)$ & \multicolumn{2}{|c|}{$1136(1271)$} & Lys-Tyr & $\alpha_{s 1}(103-114)$ \\
\hline & 1162 & $1033(1168)$ & \multicolumn{2}{|c|}{$904(1039)$} & Glu-Glu & $\alpha_{s 2}(156-164)$ \\
\hline & 913 & 784 & \multicolumn{2}{|l|}{628} & Glu-Arg & $\alpha_{\mathrm{sl}}(89-95)$ \\
\hline & 865 & 778 & \multicolumn{2}{|l|}{691} & Ser-Ser & $\beta(17-22)$ \\
\hline & 806 & 659 & \multicolumn{2}{|l|}{560} & Phe-Val & $\alpha_{\mathrm{s} 1}(24-30)$ \\
\hline & 771 & 642 & \multicolumn{2}{|l|}{514} & Glu-Gln & $\alpha_{\mathrm{s} 1}(96-101)$ \\
\hline \multirow[t]{3}{*}{18} & 1810 & 1681 & \multicolumn{2}{|l|}{1610} & Glu-Ala & $\alpha_{s 1}(61-74) 4 P$ \\
\hline & 905 & 791 & \multicolumn{2}{|l|}{662} & Asn-Glu & $\alpha_{s 1}(17-23)$ \\
\hline & 791 & 662 & \multicolumn{2}{|l|}{548} & Glu-Asn & $\alpha_{\mathrm{s1}}(18-23)$ \\
\hline \multirow[t]{6}{*}{19} & 1340 & 1253 & \multicolumn{2}{|l|}{1140} & Ser-Leu & $\beta(57-68)$ \\
\hline & 1251 & $1150(1285)$ & \multicolumn{2}{|l|}{1022} & Thr-Lys & $\alpha_{s 2}(198-207)$ \\
\hline & 1219 & 1120 & \multicolumn{2}{|l|}{1049} & Val-Ala & $\alpha_{\mathrm{s} 1}(25-35)$ \\
\hline & 1090 & $991(1126)$ & \multicolumn{2}{|l|}{920} & Val-Ala & $\alpha_{s 1}(25-34)$ \\
\hline & 983 & 905 & 791 & 662 & Asn-Glu & $\alpha_{\mathrm{s} 1}(17-23)$ \\
\hline & 735 & 622 & \multicolumn{2}{|l|}{523} & Leu-Val & $\alpha_{\mathrm{s} 1}(58-63)$ \\
\hline \multirow[t]{6}{*}{20} & 1765 & 1678 & 1565 & & Ser-Leu & $\beta(57-72)$ \\
\hline & 1678 & 1565 & 1466 & & Leu-Val & $\beta(58-72)$ \\
\hline & 1494 & 1423 & 1292 & & Ala-Met & $\alpha_{\mathrm{s} 1}(53-65)$ \\
\hline & 1283 & 1154 & 998 & & Glu-Arg & $\alpha_{\mathrm{s} 1}(89-98)$ \\
\hline & 1117 & 1018 & 905 & & Val-Leu & $\alpha_{\mathrm{s} 1}(15-23)$ \\
\hline & 1022 & 923 & 810 & & Val-Ile & $\alpha_{\mathrm{s} 2}(200-207)$ \\
\hline 21 & 2186 & 2099 & 1986 & 1887 & Ser-Leu-Val & $\beta(57-76)$ \\
\hline & 2099 & 1986 & 1887 & 1724 & Leu-Val-Tyr & $\beta(58-76)$ \\
\hline & 1949 & 1802(1937) & 1838 & 1767 & Phe-Val-Ala & $\alpha_{\mathrm{s} 1}(24-40)$ \\
\hline & 1725 & 1638 & 1525 & 1426 & Ser-Leu-Val & $\beta\left(57-72\right.$, Pro $\left.^{67}\right)$ \\
\hline & 1551 & 1450 & 1322 & 1221 & Thr-Gln-Thr & $\beta(78-91)$ \\
\hline & 1366 & $1219(1354)$ & 1120 & 1049 & Phe-Val-Ala & $\alpha_{\mathrm{s} 1}(24-35)$ \\
\hline & 1237 & $1090(1225)$ & 991 & 920 & Phe-Val-Ala & $\alpha_{s 1}(24-34)$ \\
\hline & 905 & 806 & 735 & 638 & Val-Ala-Pro & $\alpha_{\mathrm{sl}}(25-32)$ \\
\hline & 770 & 671 & 572 & 473 & Val-Val-Val & $\beta(82-88)$ \\
\hline 22 & 1854 & 1707 & 1560 & & Phe-Phe & $\alpha_{\mathrm{s} 1}(23-38)$ \\
\hline & 1641 & 1494 & 1347 & & Phe-Phe & $\alpha_{\mathrm{s} 1}(23-36)$ \\
\hline & 1052 & 905 & 806 & & Phe-Val & $\alpha_{\mathrm{s} 1}(24-32)$ \\
\hline & 809 & 695 & 582 & & Asn-Leu & $\alpha_{s 1}(19-24)$ \\
\hline 23 & 2510 & 2423 & 2310 & & Ser-Leu & $\beta(69-91)$ \\
\hline & 2299 & 2212 & 2099 & & Ser-Leu & $\beta(57-77)$ \\
\hline & 2043 & 1956 & 1843 & & Ser-Leu & $\beta(69-87)$ \\
\hline & 1880 & 1717 & 1589 & & Tyr-Gln & $\beta(193-209)$ \\
\hline & 1781 & 1680 & 1552 & & Thr-Gln & $\beta(78-93)$ \\
\hline & 1717 & 1589 & 1460 & & Gln-Glu & $\beta(194-209)$ \\
\hline & 1641 & 1584 & 1471 & & Gly-Leu & $\alpha_{s 1}(10-23)$ \\
\hline & 1522 & 1423 & 1267 & & Glu-Arg & $\alpha_{\mathrm{s} 1}(89-100)$ \\
\hline & 1396 & 1267 & 1111 & & Glu-Arg & $\alpha_{s 1}(89-99)$ \\
\hline & 1052 & 905 & 806 & & Phe-Val & $\alpha_{\mathrm{s} 1}(24-32)$ \\
\hline 24 & 2740 & 2653 & 2540 & & Ser-Leu & $\beta(69-93)$ \\
\hline & 2316 & 2201 & & & Asp- & $\kappa(20-28)$ \\
\hline & 1700 & 1700 & & & Gln-Glu & $\beta(194-209) *$ \\
\hline
\end{tabular}

${ }^{*} \mathrm{Gln}{ }^{194}$ cyclyzed to pyroglutamic acid. Figures of $\mathrm{MH}^{+}$in parentheses represent a 135 -molecular mass increase due to the phenylisothiocyanate coupling with the $\varepsilon-\mathrm{NH}_{2}$ group of each lysine residue.

* Gln ${ }^{194}$ cyclisée en acide pyroglutamique. Les valeurs de $\mathrm{MH}^{+}$entre parenthèses représentent une augmentation de 135 unités de masse, due à la fixation de phenylisothiocyanate sur les groupement $\varepsilon-\mathrm{NH}_{2}$ de chaque résidu lysine. 
molecular mass lower than $3 \mathrm{kDa}$ were identified in the 14-month-old GP cheese sample as shown in table II. It was not possible to record mass values for the material recovered from HPLC separation before peak 1 (fig 1a).

Thirty-two peptides originating from $\beta$-, 44 from $\alpha_{\mathrm{s} 1^{-}}, 13$ from $\alpha_{\mathrm{s} 2}-\mathrm{CN}$, and three from para- $\mathrm{K}-\mathrm{CN}$, were readily identified by using this procedure.

\section{Peptides in 38-month-old Grana Padano cheese}

The HPLC peaks of the 38-month-old cheese sample (fig 1b) were collected single or in groups and the relative peptide components identified by the same analytical procedure indicated above. In table III the list of the peptide components occurring in the 38-month-old GP cheese sample is reported.

Eight peptides were from $\beta$-, twentyseven from $\alpha_{\mathrm{s} 1^{-}}$, one from $\alpha_{\mathrm{s} 2^{-}}$, and two from para- $\mathrm{K}-\mathrm{CN}$. Some peptide components were common to both the younger and the older cheese. The 38-month-old GP cheese sample contained $15 \alpha_{s 1}{ }^{-}$, five $\beta$ - novel peptides, and one $\kappa-\mathrm{CN}$-novel peptide. It must be underlined that the number of peptides of the aged cheese was apparently lower than that of the younger cheese, and that the peptides from the older cheese were generally shorter forms of the components detected in the younger cheese.

In the 38-month-old cheese sample only one precursor of the $\beta-\mathrm{CM} 7$ was detected, the $\beta-\mathrm{CN}(\mathrm{f} 60-72)$ component, which means that no one of the seven bioactive-precursor peptides containing the sequence $60-66$ detected in the 14-month-old cheese resisted to the cheese enzymes. Therefore, one might consider that peptide $60-72$ is the terminal product of all the $\beta$-CM precursors of the 14-month-old cheese.

Seven closely-related $\alpha_{\mathrm{s} 1}$-CN-CPPs differing either for the $\mathrm{N}$-terminal extension
$63 / 64 / 65 / 67-74$ or the level of phosphorylation eg 4P to IP were found (table III).

Together with (61-74)4P and (62-74)4P peptides, the same components were found in a CPP fraction specifically isolated from the $\mathrm{pH} 4.6$ soluble fraction of the 14-monthold cheese sample (P Ferranti, personal communication). However, the mixture lacked the less phosphorylated (65-74)3P peptide which was found only in the 38-month-old cheese sample. N-terminal Ser ${ }^{115}$ was found either phosphorylated or unphosphorylated.

\section{Identification of peptides with molecular mass above $10 \mathrm{kDa}$ in the 14-month-old Grana Padano cheese}

A preparative fractionation of the peptides having a molecular mass above $10 \mathrm{kDa}$ carried out by chromatography on MonoQ column showed the profile which in figure 2 is similar to that previously obtained for a 24-month PR cheese sample (Addeo et al, 1995). Here also, the protein material eluted under each chromatographic peak was a mixture of components. As example, the last peak eluted from the MonoQ column analyzed by gel electrophoresis at alkaline $\mathrm{pH}$ presented three main bands (fig 3), $\alpha_{\mathrm{s1}}{ }^{-}$ $\mathrm{CN}$ being the most important whereas $\alpha_{\mathrm{s} 1}$ $\mathrm{I}-\mathrm{CN}$ and unidentified components in lower amounts. In order to further fractionate this material, a HPLC chromatography was carried out on a $\mathrm{C} 4$ column, as shown in figure 4 . Thirteen peaks were collected which were submitted to analysis by ES/MS. Since each peak was constituted by a mixture of components, a combination of ES/MS for the molecular mass determination and manual Edman degradation for the N-terminal sequence determination was used for the identification of the HMM-peptides. The determination of at least two N-terminal residues together with the molecular mass of the native peptides was necessary to unambiguously identify HMM-peptides in mix- 
Table III. Oligopeptide components from a 38-month-old Grana Padano cheese found in the fractions separated by HPLC (fig 1b).

Peptides provenant de la fraction de masse moléculaire inférieure à $3 \mathrm{kDa}$ d'un Grana Padano de 38 mois identifiés dans les fractions obtenues en CLHP (fig $\mathrm{lb}$ ).

\begin{tabular}{|c|c|c|c|c|c|c|}
\hline \multirow[b]{2}{*}{$\begin{array}{l}\text { HPLC } \\
\text { peak }\end{array}$} & \multirow[b]{2}{*}{$M H^{+}$} & \multicolumn{3}{|c|}{$\begin{array}{l}\mathrm{MH}^{+} \text {after Edman } \\
\text { cycle }\end{array}$} & \multirow[b]{2}{*}{$\begin{array}{l}N \text {-terminal } \\
\text { sequence }\end{array}$} & \multirow[b]{2}{*}{ Peptide } \\
\hline & & $I$ & II & III & & \\
\hline \multirow[t]{3}{*}{1} & 921 & 754 & 683 & & SerP-Ala & $\alpha_{\mathrm{s} 1}(115-121) 1 \mathrm{P}$ \\
\hline & 841 & 712 & 599 & & Glu-Leu & $\alpha_{s 1}(39-45) 1 P$ \\
\hline & 589 & 460 & 36 & & Glu-Val & $\alpha_{s 2}(68-72)$ \\
\hline \multirow[t]{6}{*}{2} & 720 & 720 & 720 & & no residue & $\beta(46-51)$ \\
\hline & 761 & 632 & 515 & & Glu-Leu & $\alpha_{s 1}(39-45)$ \\
\hline & 763 & 634 & 519 & & Glu-Asp & $\alpha_{s 1}(55-60)$ \\
\hline & 954 & 867 & 700 & & Ser-SerP & $\alpha_{s 1}(64-74) 1 P$ \\
\hline & 1016 & $917(1052)$ & 93 & & Val-Leu & $\kappa(78-87)$ \\
\hline & 1034 & 867 & 700 & & SerP-SerP & $\alpha_{\mathrm{s} 1}(67-74) 2 \mathrm{P}$ \\
\hline 3 & 731 & 602 & 47 & & Glu-Lys & $\alpha_{\mathrm{s} 1}(35-40)$ \\
\hline \multirow[t]{5}{*}{4} & 1610 & 1481 & 131 & & Glu-SerP & $\alpha_{\mathrm{s} 1}(63-74) 4 \mathrm{P}$ \\
\hline & 1481 & 1314 & 120 & & SerP-Ile & $\alpha_{s 1}(64-74) 4 P$ \\
\hline & 1314 & 1201 & 103 & & Ile-SerP & $\alpha_{\mathrm{s} 1}(65-74) 3 \mathrm{P}$ \\
\hline & 1244 & 1187 & 108 & 223) & Gly-Val & $\beta(94-105)$ \\
\hline & 1187 & 1100 & 102 & & Ser-Ala & $\alpha_{s 1}(115-124)$ \\
\hline \multirow[t]{5}{*}{5} & 758 & 644 & 515 & & Asn-Glu & $\alpha_{s 1}(17-22)$ \\
\hline & 995 & $908(1043)$ & 780 & & Ser-Lys & $\beta(168-176)$ \\
\hline & 1139 & 972 & 901 & & SerP-Ala & $\alpha_{\mathrm{s} 1}(115-123) 1 \mathrm{P}$ \\
\hline & 1401 & 1314 & 120 & & Ser-Ile & $\alpha_{s 1}(64-74) 3 P$ \\
\hline & 1530 & 1401 & 131 & & Glu-Ser & $\alpha_{s 1}(63-74) 3 P$ \\
\hline \multirow[t]{2}{*}{6} & 1427 & $1299(1434)$ & 113 & & Lys-Tyr & $\alpha_{s 1}(103-114)$ \\
\hline & 806 & 659 & 560 & & Phe-Val & $\alpha_{s 1}(24-30)$ \\
\hline \multirow[t]{5}{*}{7} & 1466 & 1303 & 120 & & Tyr-Pro & $\beta(60-72)$ \\
\hline & 1299 & $1136(1271)$ & 100 & & Tyr-Lys & $\alpha_{s 1}(104-114)$ \\
\hline & 905 & 791 & 66 & & Asn-Glu & $\alpha_{\mathrm{s1}}(17-23)$ \\
\hline & 791 & 662 & 558 & & Glu-Asn & $\alpha_{s 1}(18-23)$ \\
\hline & 489 & 376 & 279 & & Leu-Pro & $\kappa(56-59)$ \\
\hline \multirow[t]{14}{*}{8} & 1022 & 923 & 810 & & Val-Ile & $\alpha_{\mathrm{s} 2}(200-207)$ \\
\hline & 1117 & 1018 & 90 & & Val-Leu & $\alpha_{s 1}(15-23)$ \\
\hline & 1237 & $1090(1225)$ & 99 & 920 & Phe-Val-Ala & $\alpha_{s 1}(24-34)$ \\
\hline & 1246 & 1117 & 1018 & & Glu-Val & $\alpha_{\mathrm{s} 1}(14-23)$ \\
\hline & 1366 & $1219(1354)$ & 1120 & 1049 & Phe-Val-Ala & $\alpha_{\mathrm{s} 1}(24-35)$ \\
\hline & 1322 & 1221 & 112 & & Thr-Pro & $\beta(80-91)$ \\
\hline & 1494 & 1347 & 1248 & & Phe-Val & $\alpha_{s 1}(24-36)$ \\
\hline & 1707 & 1560 & 146 & & Phe-Val & $\alpha_{s 1}(24-38)$ \\
\hline & 1396 & 1267 & 111 & & Glu-Arg & $\alpha_{\mathrm{s} 1}(89-99)$ \\
\hline & 1641 & 1584 & 147 & & Gly-Leu & $\alpha_{s 1}(10-23)$ \\
\hline & 1717 & 1589 & 146 & & Gln-Glu & $\beta(194-209)$ \\
\hline & 1680 & 1552 & 145 & & Gln-Thr & $\beta(79-93)$ \\
\hline & 1880 & 1717 & 158 & & Tyr-Gln & $\beta(193-209)$ \\
\hline & 1552 & 1423 & 126 & & Glu-Arg & $\alpha_{s 1}(89-100)$ \\
\hline
\end{tabular}

Figures of $\mathrm{MH}^{+}$in parenthesis represent a 135 -molecular mass increase due to the phenylisothiocyanate coupling with the $\varepsilon-\mathrm{NH}_{2}$ group of each lysine residue of peptides.

Les valeurs de $\mathrm{MH}^{+}$entre parenthèses représentent une augmentation de 135 unités de masse, due à la fixation de phenylisothiocyanate sur les groupements $\varepsilon-\mathrm{NH}_{2}$ de chaque résidu lysine des peptides. 


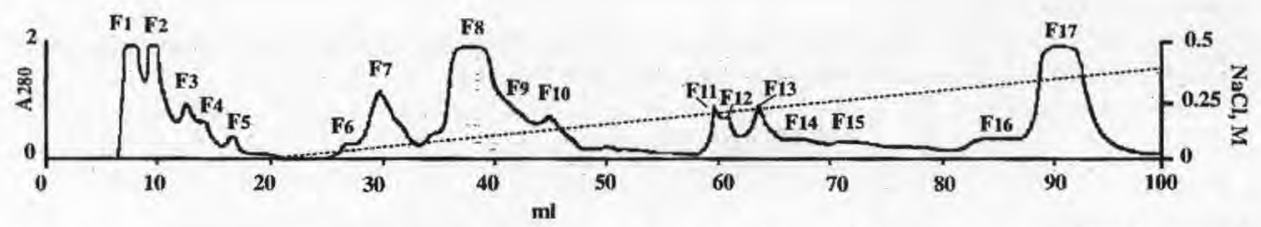

Fig 2. Chromatographic separation by FPLC on Mono Q column of the retentate on $10 \mathrm{kDa}$ cut-off membrane $(100 \mathrm{mg}$ ) of 14 -month-old Grana Padano cheese using a linear gradient of $0-0.32 \mathrm{~mol} / \mathrm{L}$ $\mathrm{NaCl}$. Fractions corresponding to each peak were pooled, dialysed against distilled water and freeze dried. Peak 17 was used both for gel electrophoretic analysis and fractionation by HPLC.

Séparation chromatographique des peptides de masse moléculaire supérieure à $10 \mathrm{kDa}(100 \mathrm{mg})$ issus d'un Grana Padano de 14 mois, sur colonne Mono $Q$ avec un gradient linéaire de 3 à 0,32 mol/L de $\mathrm{NaCl}$. Les fractions correspondant à chaque pic ont été rassemblées, dialysées contre de l'eau distillée et lyophilisées. Le pic 17 a été utilisé pour l'électrophorèse et le fractionnement par CLHP.

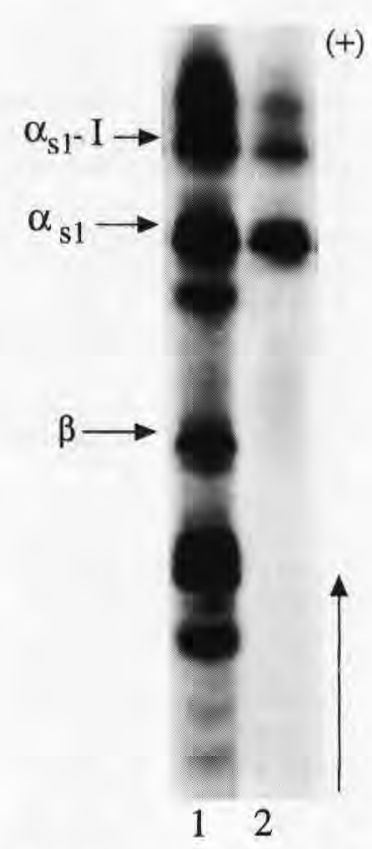

Fig 3. Polyacrylamide gel disc-electrophoresis at $\mathrm{pH} 8.6$ of the retentate on a $10-\mathrm{kDa}$ cut-off membrane (fraction B) from a 14-month-old Grana Padano cheese (lane 1) and the most retained protein components on the MonoQ column (lane 2).

Électrophorèse en gel de polyacrylamide à pH 8,6 des peptides de masse moléculaire supérieure à $10 \mathrm{kDa}$ (fraction B) issus d'un Grana Padano de 14 mois (puits 1) et de la fraction protéique la plus retenue sur colonne MonoQ (puits 2). ture. The peptides identified in the seven HPLC peaks (7 to 13) are reported (table IV).

The high number of components identified means that neither electrophoresis nor HPLC was able to reveal the complexity of the casein composition as ES/MS did. A number of protein species was found consisting of either intact $\alpha_{\mathrm{s}^{-}}, \alpha_{\mathrm{s} 2^{-}}$, and $\beta-\mathrm{CN}$ and of derived peptides. Native $\alpha_{\mathrm{s} 1}-\mathrm{CN} B$ genetic variant was found to be a more complex mixture of differently phosphorylated protein chains containing 5 to $9 \mathrm{P}$ instead of just two phosphorylation levels, eg, 8 and $9 \mathrm{P}$, as reported in literature (Swaisgood, 1993). Only the $\alpha_{\mathrm{s} 1}$-CN C-8P form was detected in HPLC fraction 9, although other differently phosphorylated forms can occur in other, not yet examined chromatographic fractions. Similarly, two $\alpha_{\mathrm{s} 2}-\mathrm{CN}(1-207) 8 \mathrm{P}$ and 9P were detected among the $\alpha_{\mathrm{s} 2}-\mathrm{CN}$ components never reported to occur in bovine casein. We do not know if they were native or new-formed partially dephosphorylated components. Surprisingly, the occurrence of $\alpha_{\mathrm{s} 1}-\mathrm{CN}$-derived peptides 24-199 and 25-199, at different levels of phosphorylation, derived from both the $\alpha_{\mathrm{s} 1}-\mathrm{CN} B$ and $\mathrm{C}$ variants, confirms the susceptibility of the 23-24 and 24-25 bonds. Both the 1-22 and 1-23 peptides were identified either in the GP cheese whey or the in vitro hydrolyzate of $\alpha_{\mathrm{s} 1}-\mathrm{CN}$ by rennet (Chianese et al, 


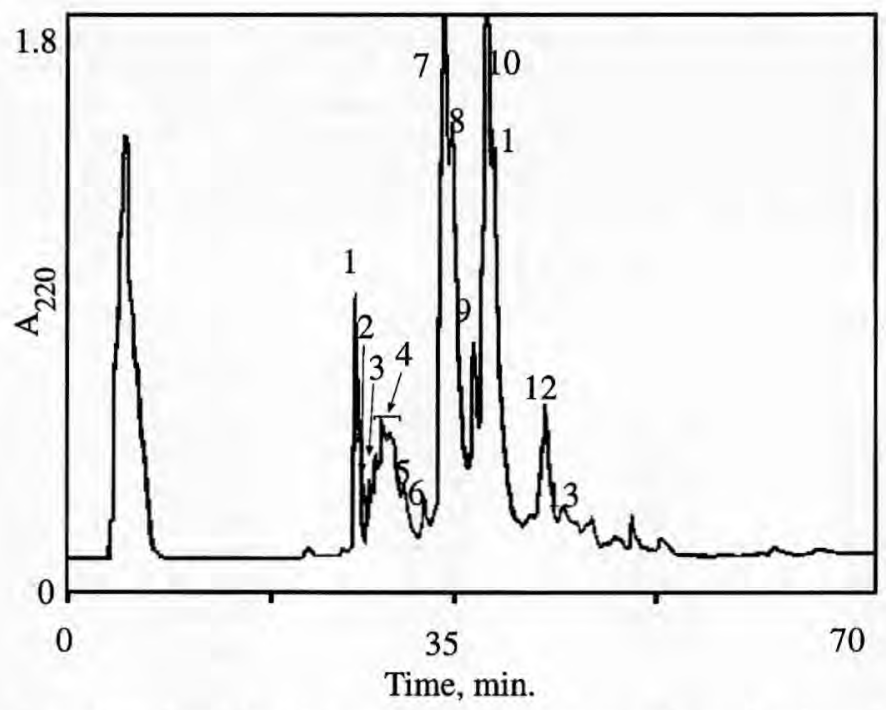

Fig 4. Reversed-phase HPLC profile of the most retained fraction on MonoQ column (lane 2, fig 3) isolated from the fraction $B$ containing peptides with a molecular mass higher than $10 \mathrm{kDa}$. C4 column; $5 \mathrm{mg}$ of peptides injected. Solvent A was $1 \mathrm{~mL}$ trifluoracetic acid/L distilled water; solvent $\mathrm{B}, 1 \mathrm{~mL}$ trifluoroacetic acid/L acetonitrile. The gradient was: $\mathrm{t}=0, \% \mathrm{~B}, 35 ; \mathrm{t}=10 \% \mathrm{~B}, 35 ; \mathrm{t}=80$, $\% \mathrm{~B}, 100 ; \mathrm{t}=90, \% \mathrm{~B}, 100 ; \mathrm{t}=90.1, \% \mathrm{~B}, 35 ; \mathrm{t}=100, \% \mathrm{~B}, 35$. The flow was maintained at $2 \mathrm{~mL} / \mathrm{min}$ and absorbance of eluate was followed at $220 \mathrm{~nm}$.

Profil CLHP en phase inverse de la fraction protéique la plus retenue sur colonne Mono $Q$ cellulose (puits 2, fig 3) issus de la fraction B, qui contient les peptides de masse moléculaire supérieure à $10 \mathrm{kDa}$. (Colonne $C 4 ; 5 \mathrm{mg}$ de peptides injectés. Tampon $\mathrm{A}$ : acide trifluoroacétique de $1 \mathrm{~mL} / \mathrm{L}$ d'eau distillée ; tampon $B$ : acide trifluoroacétique à $1 \mathrm{~mL} / \mathrm{L}$ d'acétonitrile. Gradient : $t=0,35 \% B$ $; t=10,35 \% B ; t=80,100 \% B ; t=90,100 \% B ; t=90,1,35 \% B ; t=100,35 \%$ B. Débit maintenu à $2 \mathrm{~mL} / \mathrm{min}$ et absorption à $220 \mathrm{~nm}$ ).

1997). One may hypothesize that the fragment 24-199 $\alpha_{\mathrm{s1}}-\mathrm{CN}$ chymosin-derived and that 25-199 was the product with the N-terminal residue-depleted by a cheese aminopeptidase. The fragment 1-143 also seems to come from the hydrolysis of $\alpha_{\mathrm{s1}}$ - $\mathrm{CN}$ by chymosin although neither Pélissier et al (1974) nor McSweeney et al (1993) reported the cleavage of the $142-143$ bond by chymo$\sin$ in solution. Similarly, the four closelyrelated $\beta$-CN peptides (f7/8/9/11-209) corresponded either to site cleavages not previously reported to be derived from endoproteases at known specificity or to intermediate transient peptides produced by cheese aminopeptidases by action on a parent HMM-peptide.

\section{DISCUSSION}

\section{An efficacious fractionation process and an advanced strategy of identification}

Singh et al (1995) pointed out that diafiltration gave very reproducible results on the $\mathrm{N}$ form separation and believed that no artifacts were formed during the fractionation procedure, given both the short time needed for the operation and the low temperature at which ultrafiltration occurs in the presence of preservative agents. Preliminary assays on three consecutively diafiltered fractions by determining identity of the HPLC peak components through FAB/MS, 
Table IV. Oligopeptides identified under the HPLC peaks 7-13, as shown in figure 4, occurred in the most retained fraction on Mono Q column (lane 2, fig 3) from fraction B containing peptides at a molecular mass higher than $10 \mathrm{kDa}$ of a 14-month-old Grana Padano cheese sample.

Peptides identifiés dans les pics CLHP 7 à 13 (fig 4) issus de la fraction la plus retenue sur la colonne Mono $Q$ (puits 2, fig 3) après injection de la fraction B, qui contient les peptides de masse moléculaire supérieure à $10 \mathrm{kDa}$, d'un échantillon de Grana Padano de 14 mois.

\begin{tabular}{|c|c|c|c|c|c|}
\hline \multirow[b]{2}{*}{$\begin{array}{l}\text { HPLC } \\
\text { peak }\end{array}$} & \multirow[b]{2}{*}{$\mathrm{MH}^{+}$} & \multicolumn{2}{|c|}{$\mathrm{MH}^{+}$after Edman } & \multirow[b]{2}{*}{$\begin{array}{l}N \text {-terminal } \\
\text { sequence }\end{array}$} & \multirow[b]{2}{*}{ Peptide } \\
\hline & & Cycle I & Cycle II & & \\
\hline \multirow[t]{5}{*}{7} & 20880 & 20735 & 20662 & Phe-Val & $\alpha_{\mathrm{s} 1} \mathrm{C}(24-199) 8 \mathrm{P}$ \\
\hline & 20787 & 20635 & 20535 & Phe-Val & $\alpha_{\mathrm{sI}}^{\mathrm{sI}} \mathrm{B}(24-199) 7 \mathrm{P}$ \\
\hline & 20733 & 20634 & 20563 & Val-Ala & $\alpha_{\mathrm{s1}} \mathrm{C}(25-199) 8 \mathrm{P}$ \\
\hline & 17213 & 17059 & 16960 & Arg-Pro & $\alpha_{\mathrm{sl}}(1-143) 8 \mathrm{P}$ \\
\hline & 17059 & 16965 & 16832 & Pro-Lys & $\alpha_{\mathrm{s1}}(2-143) 8 \mathrm{P}$ \\
\hline \multirow[t]{3}{*}{8} & 20732 & 20633 & 20561 & Val-Ala & $\alpha_{\mathrm{sl}} \mathrm{B}(25-199) 8 \mathrm{P}$ \\
\hline & 20782 & 20638 & 20536 & Phe-Val & $\alpha^{\mathrm{si}} \mathrm{B}(24-199) 7 \mathrm{P}$ \\
\hline & 20876 & 20729 & 20657 & Phe-Val & $\alpha_{\mathrm{sl}} \mathrm{C}(24-199) 8 \mathrm{P}$ \\
\hline 9 & 23685 & nd & & Arg-Pro & $\alpha_{\mathrm{s} 1} \mathrm{C} 8 \mathrm{P}$ \\
\hline \multirow[t]{2}{*}{10} & 23611 & 23451 & 23362 & Arg-Pro & $\alpha_{\mathrm{s} 1} \mathrm{~B}(1-199) 8 \mathrm{P}$ \\
\hline & 23531 & 23378 & 23281 & Arg-Pro & $\alpha_{s 1} B(1-199) 7 P$ \\
\hline \multirow[t]{2}{*}{11} & 23611 & 23458 & 23361 & Arg-Pro & $\alpha_{s 1} B(1-199) 8 \mathrm{P}$ \\
\hline & 23531 & 23375 & 23278 & Arg-Pro & $\alpha_{\mathrm{s} 1} \mathrm{~B}(1-199) 7 \mathrm{P}$ \\
\hline \multirow[t]{6}{*}{12} & 25100 & nd & & & $\alpha_{\mathrm{s} 2} 9 \mathrm{P}$ \\
\hline & 25020 & nd & & & $\alpha_{\mathrm{s} 2}^{\mathrm{s} 2 \mathrm{P}}$ \\
\hline & 22438 & nd & & Arg-Pro & $\alpha_{\mathrm{s} 1} \mathrm{~B} 6 \mathrm{P}$ \\
\hline & 22358 & nd & & Arg-Pro & $\alpha_{s 1} B 5 P$ \\
\hline & 22845 & nd & & & $\beta \mathrm{A}^{2}(11-207)$ \\
\hline & 23116 & nd & & & $\beta A^{2}(7-207)$ \\
\hline \multirow[t]{3}{*}{13} & 23989 & nd & & Arg-Glu & $\beta A^{2} 5 \mathrm{P}$ \\
\hline & 24574 & nd & & & $\alpha_{s 2}(9-209)$ \\
\hline & 24744 & nd & & & $\alpha_{\mathrm{s} 2}(8-209)$ \\
\hline
\end{tabular}

nd, not determined.

nd, non déterminé.

did not show any processing of our peptides. However, as pointed out by Singh et al (1995), hydrophilic peptides can accumulate in the permeate as the polysulphone membranes tend to reject hydrophobic peptides. Such a mechanism was actually observed by us during diafiltration of in vitro hydrolysate of whole casein by rennet. Peptides such as $\alpha_{s 1}$ (f1-22) and $\alpha_{s 1}(f 1-23)$ having both a nominal molecular mass below $3000 \mathrm{kDa}$, eg, 2617 and 2764, respectively were found to concentrate in the retentate (Chianese et al, 1997). This means that these hydrophobic oligopeptides would accumulate in the fraction containing components at molecular mass in the intermediate range between 3 and $10 \mathrm{kDa}$, not analyzed within this work. Concerning the strategy of peptide identification, the use of FAB/MS and ES/MS has allowed us to directly analyze peptides, without any necessary derivatization reaction, which represents an advance in comparison with the old time-consuming procedures requiring a preliminary derivatization step. However, the molecular mass determination alone was insufficient to allow the identification of peptides. For this, it was necessary to submit 
the peptide mixture to a manual Edman degradation step followed by FAB/ or ES/MS re-examination of the truncated peptide mixture. The mass signals shifted according to the mass value of the $\mathrm{N}$-terminal residue and this procedure repeated once or twice allowed to identify unambiguously the peptides. Since a partial suppression of the signals taking place in FAB analysis (Naylor et al, 1986), having as upper detection limit about $3000 \mathrm{Da}, \mathrm{ES} / \mathrm{MS}$ was used, especially for large peptides and proteins. These techniques were of utmost importance for identifying cheese protein and peptides characterized by a microheterogeneity such as the discrete phosphorylation and the simultaneous presence of closely related differently long peptides.

\section{The mechanisms of casein degradation in Grana Padano cheese}

The proposed $\beta-\mathrm{CN}$ derived peptides identified in the 14-month-old GP cheese sample, originated mainly from the $\mathrm{N}$-terminal half protein, perhaps from the soluble peptides 1-105/107 (the protease-peptones complementary to $\gamma^{2}$ - and $\gamma^{3}-\mathrm{CN}$ ). There were five components coming from the $\beta-\mathrm{CN}(\mathrm{fl} 1-28) 4 \mathrm{P}$-plasmin mediated peptide, 23 from the peptide region between 44 and 100 residues and two from the $\mathrm{C}$-terminal region of $\beta-C N$ as a consequence of the peptide bond cleavage between residues $192 / 193$ and $193 / 194$. These last two are cleavage sites of the Lactococcus cell envelope proteinase (Singh et al, 1995). The formation of soluble peptides from the $\mathrm{N}$-terminal region of $\beta-\mathrm{CN}$ is consistent with our previous results showing an increase of $\gamma$ - $\mathrm{CN}$ during GP ripening (Addeo et al, 1996). This increase is linked to a parallel increase of the soluble proteose-peptones such as 1-28, 1-105, 1-107, 29-105, and 29-107 from the plasmin action on $\beta-C N$ (Andrews, 1978a, b).
Two peptides, differing only for the amino acid substitution $\mathrm{His}^{67} \rightarrow \mathrm{Pro}^{67}$ were detected, which indicated that cheese milk consisted of a mixture of $\beta-\mathrm{CN}$ variants such as $\mathrm{A}^{1}, \mathrm{~B}$, and $\mathrm{C}$ and $\mathrm{A}^{2}$ and $\mathrm{A}^{3}$, having $\mathrm{His}^{67}$ and $\mathrm{Pro}^{67}$ as marker-residues respectively. The amino acid sequence $60-66$ corresponding to the $\beta$-casomorphin 7 ( $\beta$-CM7) (Brantl, 1979) was found in eight peptides which represent actual precursors of bioactive peptides. No peptide containing residues between 23 and 43 was isolated from the 14-month-old cheese. This means that, if formed, these peptides were degraded by the cheese exopeptidases into free amino acids within the first months of ripening. An aminopeptidase activity was clearly deduced from the simultaneous occurrence in the cheese of four peptides in addition to the parent $\beta-\mathrm{CN}(44-52)$, having one to three $\mathrm{N}$-terminal amino acid residues less. Similarly, pairs of peptides such as $1-6$ and 2-6, 58-77 and 57-77, 58-76 and 57-76 are to be considered also the degradation result of an active aminopeptidase which removes the $\mathrm{N}$-terminal residues, one by one, from the parent peptides. The pair of peptides 58-77 and 58-76, 44-52 and 44-51 also seems indicative of a carboxypeptidase activity in cheese which is known to remove one by one the C-terminal residues of peptides. Finally, the two pairs of peptides $78-93$ and 78-91 and 69-93 and 69-91, were indicative of a possible oligoendopeptidase activity converting parent peptides into two-residue shortened at the C-terminal end.

Among the $44 \alpha_{\mathrm{s} 1}-\mathrm{CN}$-derived peptides of the 14-month-old GP cheese sample, 30 were from the 1-79 region, and the others from the peptide bond cleavage within the residues 89 and 166 . No peptide started with a residue beyond this site, meaning that the $\alpha_{s 1}$-CN(f1-79) component, formerly named $\alpha_{\mathrm{s} 1}$-PL1, which originated in cheese by the plasmin action on $\alpha_{\mathrm{s} 1}-\mathrm{CN}$ (Addeo et al, 1995), could be the parent peptide. From this soluble peptide, by action of cheese 
endopeptidases, a number of shorter peptides could take origin further processed by cheese exopeptidases. This was also verified by examining the $10-16 / 17 / 18$ and $19-23 / 24$ and $15 / 17 / 18-23$, and $17-22$ and 14-22, which differed for one or more $\mathrm{N}$ - and/or C-terminal amino acid residues. An additional set of peptides consisting of the components 24-40, 24-38, 24-36, $24-35,25-35$ and $24-34$ and $25-34$ and 24-32 and $25-32$ and $24-30$ is also a complex mixture of peptides formed from an unknown parent peptide which was degraded by cheese endopeptidases and exopeptidases into a number of individual components differing by one or more amino acid residues.

Of the $13 \alpha_{\mathrm{s} 2}$ - CN peptides identified in the 14-month-old GP cheese sample, ten originated from the C-terminal region. The components constituting the peptide set 170/172/173-178, 175-182, 177-180, and 179-184 were closely-related to each other, indicating a common origin from a parent soluble peptide. However, the peptides 198-205, 198-207 and 200-207 could originate from the chymosin activity on $\alpha_{\mathrm{s} 2}$-CN since both the components $\alpha_{s 2}(f 198-207)$ and $\alpha_{s 2}(f 200-207)$ were generated, among other peptides, during the in vitro action of rennet on whole casein (Chianese et al, 1997). Only three peptides were isolated from the para- $\kappa$-casein part, indicating that this peptide was also sensitive to protease attack even if to a lesser extent than the other casein fractions.

Although only two samples of different ages were analyzed in detail, the changes occurring in the oligopeptide fraction of the 14 and 38-month-old cheese samples gave some precise indications on the production of peptides. The $\alpha_{s 1}$ - $\mathrm{CN}$-derived peptides of the permeate containing peptides molecular mass with than $3 \mathrm{kDa}$ dominated, both in young and old GP cheese, over those derived from $\beta-\mathrm{CN}$. This was consistent with the results of previous studies concerning the degradation of $\beta$ - and $\alpha_{s 1}$-casein which continued throughout the ripening process (Addeo et al, 1996) by action of plasmin mainly. After about 12 months, $\beta$-casein was entirely hydrolyzed whereas about $50 \%$ initial $\alpha_{s 1}$-casein residue in cheese (Addeo et al, 1996). Thus, the contribution of $\beta$ - and $\alpha_{s 1}$-casein to form oligopeptides in GP cheese was unequal, the former being more important. Some $\beta$-CN-derived peptides might be also more susceptible to exopeptidases than the $\alpha_{\mathrm{s1}}-\mathrm{CN}$ peptides becoming hydrolyzed in mature cheese. Therefore, a degradation scheme of peptides in GP cheese necessarily includes a concerted action of endopeptidases, and exopeptidases. Soluble peptides are the result of the intervention: of i) endopeptidases such as plasmin and others from bacterial origin; and ii) aminopeptidases, and carboxypeptidases, both removing one by one amino acid residues from the terminal ends of soluble peptides. Important enzyme activities are those associated with the cell-envelope or the cytoplasm of $L$ helveticus species dominating in GP cheese. These enzymes seem to produce a casein hydrolysate containing flavor-significant oligopeptides from which free amino acids, particularly glutamate, the most represented in GP cheese, giving a brothy taste to mature GP cheese, are formed.

\section{ACKNOWLEDGMENT}

This work was supported by the Italian National Research Council (CNR), RAISA special project, sub-project No 4 .

\section{REFERENCES}

Addeo F, Chianese L, Salzano A, Sacchi R, Cappuccio U, Ferranti P, Malorni A (1992) Characterization of the $12 \%$ trichloroacetic acid-insoluble oligopeptides of Parmigiano Reggiano cheese. J Dairy Res $59,401-411$ 
Addeo F, Chianese L, Sacchi R, Spagna Musso S, Ferranti P, Malorni A (1994) Characterization of oligopeptides of Parmigiano-Reggiano cheese soluble in $120 \mathrm{~g}$ trichloroacetic/L.J Dairy Res 61 365-374

Addeo F, Garro G, Intorcia N, Pellegrino L, Resmini P, Chianese L (1995) Gel electrophoresis and immunoblotting for the detection of casein proteolysis in cheese. J Dairy Res 62, 297-309

Addeo F, Chianese L, Intorcia N, Petrosino R, Cappuccio U, Garro G, Hogenboom J (1996) Aspetti caratteristici della proteolisi nel formaggio Grana Padano. In: Grana Padano, un formaggio di qualità. (Consorzio per la tutela del formaggio Grana Padano, ed) Milano, 61-98

Andrews AT (1978a) The composition, structure and origin of proteose-peptone component 5 of bovine milk. Eur J Biochem 90, 59-65

Andrews AT (1978b) The composition, structure and origin of proteose-peptone component $8 \mathrm{~F}$ of bovine milk. Eur J Biochem 90, 67-7j

Bottazzi V, Battistotti B, Cucchetti G (1996) Aspetti caratteristici della proteolisi nel formaggio Grana Padano. In: Grana Padano, un formaggio di qualità. (Consorzio per la tutela del formaggio Grana Padano, ed). Milano, 49-59

Brantl V, Teschemacher H, Henschen A, Lottspeich F (1979) Novel opioid peptides derived from casein ( $\beta$-Casomorphins): I. Isolation from bovine casein peptone. Hoppe Seyler's Z Physiol Chem. 360 1211-1216

Chianese L, Caira S, Ferranti P, Laezza P, Malorni A, Mucchetti G, Garro G, Addeo F (1997) The oligopeptides of sweet and acid cheese whey. Lait, 77, 693-709

Ferranti P, Malorni A, Nitti G, Laezza P, Pizzano R, Chianese L, Addeo F (1995) Primary structure of ovine $\alpha_{\mathrm{s}}$-caseins: localization of phosphorylation sites and characterization of genetic variants A,C, and D. J Dairy Res 62, 281-296

Ferranti P, Barone F, Chianese L, Addeo F, Scaloni A, Pellegrino L, Resmini P (1997) Phosphopeptides from Grana Padano cheese: nature, origin, and changes during ripening. $J$ Dairy Res, submitted

Fox PF, McSweeney PLH (1994) Applications of chromatography in the study of cheese ripening and quality. In: Relating Instrumental, Sensory and
Consumer Data. EC FLAIR SENS publication series, Vol 3, (Management Team of FLAIR SENS project, ed), MATFORSK, $1430 \AA$ A (Norway). $25-59$

Grappin R, Rank TC, Olson NF (1985) Primary proteolysis of cheese proteins during ripening. J Dairy Sci $68,531-540$

Khalid NM, EI Soda M, Marth EH (1991) Peptide hydrolases of Lactobacillus helveticus and Lactobacillus delbrueckii spp bulgaricus. J Dairy Sci $74,29-45$

Le Bars D, Gripon JC (1989) Specificity of plasmin towards bovine $\alpha_{s 2}$-casein. J Dairy Res $56,817-821$

Le Bars D, Gripon JC (1993) Hydrolysis of $\alpha_{\mathrm{s} 1}$-casein by plasmin. Lait 73, 337-344

McSweeney PLH, Olson NF, Fox PF, Healy A, Højrup P (1993) Proteolytic specificity of chymosin on $\alpha_{\mathrm{s} 1}$-casein. J Dairy Res 60, 401-412

Naylor S, Findeis AF, Gibson BW, Williams DH (1986) An approach toward the complete fast atom bombardment analysis of enzymatic digests of peptides and proteins. J Am Chem Soc 108, 6359-6363

Pélissier JP, Mercier JC, Ribadeau Dumas B (1974) Etude de la protéolyse des caséines $\alpha_{\mathrm{s} 1}$ et $\beta$ par la présure. Spécificité d'action. Peptides amers libérés. Ann Biol Anim Bioch Biophys 14, 343-363

Pritchard GG, Colbear T (1993) The physiology and biochemistry of the proteolytic system in lactic acid bacteria. FEMS Microbiol Rev 12 179-206

Roudot-Algaron F, Le Bars D, Kerhoas L, Einhorn J, Gripon JC (1994) Phosphopeptides from Comté cheese: nature and origin. J Food Sci 59, 544-547

Singh TJ, Fox PF, Healy Á (1995) Water-soluble peptides in Cheddar cheese: isolation and identification of peptides in the diafiltration retentate of the water-soluble fraction. J Dairy Res 62 629-640

Swaisgood H (1993) Chemistry of caseins. In: Advanced Dairy Chemistry. Vol 1. Proteins (P Fox, ed) Elsevier Applied Science, London, 63-110

Visser S (1993) Proteolytic enzymes and their action on milk proteins. A review. Neth Milk Dairy $J 35$, 65-88

Zevaco C, Gripon JC (1988) Properties and specificity of a cell-wall proteinase from Lactobacillus helveticus. Lait $68,393-408$ 